\title{
Microfluidic Microchannel (Size And Shape) for Single Cell Analysis by Numerical Optimization: Lateral Trapping Method
}

\author{
Sushmitha.M, Jasper James.A, Vigneswaran Narayanamurthy, Padmasini.N, Fahmi Samsuri
}

\begin{abstract}
The primary objective of this work is to show simulation outputs from the developed model of cell flow within a microfluidic device. This work is essential because it requires computational models to offer compact sized biomedical equipment that involves microfluidics technology. Microfluidics has become a common technology for life science applications in latest years. The purpose is to learn the effect of various microchannel size and shape with lateral traps for single cell analysis and to arrive at an optimum design based on a simulation study using COMSOL Multiphysics software. Thus in order to develop software model of various microchannels which execute fluid flow in the microelectronic device. This research provides numerical alternatives from finite element analysis simulation using the software COMSOL-Multiphysics to characterize the shape and size of the microchannel initializing the fluid flow. Optimized design analysis and operating conditions for efficient single cell trap is reported.
\end{abstract}

Keywords: COMSOL, microchannel, microfluidics, simulation, single cell, trapping.

\section{INTRODUCTION}

$\mathrm{S}_{\text {ingle cell analysis relates to the research of individual }}$ tissue-isolated cells in multi-cellular organisms in the field of cell biology. Analysis of single cells is a natural way of studying organisms. Genetic behaviors and protein patterns can mostly elucidate cellular activities, but the complex analyses of cells helps to gain knowledge about the continuously occurring molecular events in each cell [1]. Every cell, even within the same sort of cell, is more or less distinct from the other. Studying each and every type of cell is therefore essential.

Revised Manuscript Received on December 16, 2019

* Correspondence Author

Sushmitha. M, Dept. of Biomedical Engineering, Kalasalingam Academy of Research and Education, Virudhunagar, Tamilnadu, India. Email: sushmitha.m@klu.ac.in

Jasper James. A, Innofutech, Chennai, Tamilnadu, India.

Vigneswaran Narayanamurthy*, 1 Faculty of Electrical and Electronic Engineering Technology, Universiti Teknikal Malaysia Melaka, 76100 Durian Tunggal, Melaka, Malaysia. 2 Faculty of Electrical and Electronics Engineering, University Malaysia Pahang Pekan, Pekan, Pahang 26600, Malaysia. 3 Centre of Excellence for Advanced Research in Fluid Flow, University Malaysia Pahang, Kuantan 26300, Malaysia. Email: vigneswaran@utem.edu.my

Padmasini. N, Dept. of Biomedical Engineering, Rajalakshmi Engineering College, Chennai Tamilnadu, India. Email: padmasini.r@rajalakshmi.edu.in

Fahmi Samsuri, Faculty of Electrical and Electronics Engineering, University Malaysia Pahang Pekan, Pekan, Pahang 26600, Malaysia. Email: fahmi@ump.edu.my

Highly recognized in bacteria, cellular heterogeneity is also increasingly recognized in eukaryotic cells. Cell cultures are expected and assumed to be homogeneous in nature, and the study of a group of cells would provide an accurate estimate of the cell activity in that culture or tissue; Researchers are highly aware of this, so single-cell analysis can be used to know about the consequential properties of differences in cells. The cells ' average response has been, and still is sometimes, found to be the response of all cells in that study. The approach to measurements of the ensemble is beginning to change. Microfluidics is a multidisciplinary field that intersects engineering, physics, chemistry, biochemistry, biotechnology, and nanotechnology with practical applications for system design in which small fluid volumes are processed to accomplish multiplexing, automation, and high-throughput screening. It is a collection of methods and processes to make equipment for exactly controlling and manipulating liquids in geometrically small channels [3]. A biochip is a miniaturized laboratory capable of sequentially performing more constant biochemical responses. These are set of micro-arrays arranged on the surface of a solid substratum and are intended to perform multiple tests at the same time to achieve greater speed and performance [5].

Chemical trapping, Dielectrophoretic trapping, Laser trapping, Magnetic trapping, Acoustic trapping are the various methods used for single cell analysis. The demerits of those methods are, in chemical trapping, cells require time to be getting attached, and deposition is permanent. There is also a problem with the choice of surface coatings. The occurrence of intense AC fields trigger heating of cells due to the ejection of certain voltage is a downside of dielectrophoresis. A common failing of laser trapping is that the amount of traps is limited by the existing laser power, as a fixed portion of each trap is essential [6]. In Magnetic trapping, the Cells are continuously open to electromagnetic force at a slight higher temperature; however, they have not been found to be harmful. Cell damage and changes in cell properties can occur in these cases [5]. And also it requires large workspace, number of technicians and quantity of sample required is more for processing explains the cellular behavior to the large extent. Human error may cause occurrences of false result and detection. Other micro fluidic techniques are constraint due to failure in designing and processing. A biochip is a miniaturized microarray

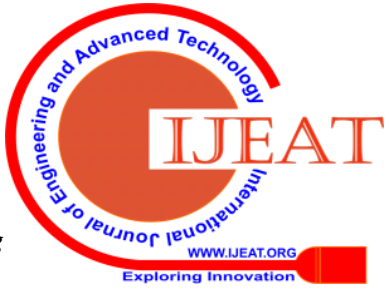


platform organized on a strong substratum that enables multiple activities to be conducted simultaneously to achieve high outcomes and speed. In this microfluidic biochip design, the whole lab work is miniaturized within a single microelectronic device. By using COMSOL Multiphysics software, the design of micro channels and modeling of the cell will be performed and the simulation results are used to validate the optimal designs [4].

Modeling of computational fluid dynamics (CFD) is an important method which is currently implemented in the field of micro level cell culture, enabling a clearer knowledge about the hydrodynamic setting and the variables that control it. CFD enables us to know the consequences of fluid flow and cell transport, thus providing essential visions into the design of microfluidic cell culture chip and its optimization [3]. CFD simulations were conducted to assess the system's characteristics during cell trapping and release [9].

This research demonstrates the design and growth of single-cell trapping using hydrodynamic cell trapping method in a microfluidic finite-element analysis [15, 19]. This work describes the design and analysis of various microchannels in microfluidic device for single cell analysis which has a wide range of application in cell engineering, protein engineering and for other diagnostic purposes [7]. Hydrodynamic trapping involves techniques for separating particles from a stream using variances in surface topography and for immobilizing them at certain places. For the most part, mechanical obstacles or hurdles are used; these only provide a passage for the fluid to filter the particles or elements from the fluid. The barrier sizes must be adjusted to the dimension of the trapped elements. Therefore, the dimensions of the barrier must be adjusted to the size of the trapped elements. Structures in which particles are stopped are vertical walls below the channel's height. Low area of shear stress is appropriate for holding elements from a running fluid. With small shear forces, feeble connections with the surface of the channel can support to retain the particle in location. There are no such rigid restrictions on particle size as filtering obstacles with this principle. For example, areas of low flow of fluid and less shear stress are developed in places manufactured in microchannel walls. Hydrodynamic trapping applications are seen in the field of drug screening or tissue engineering [16, 17]. Patch clamping on a chip is an exact example which consists of holes in the design that sucks cells on. The advantages of hydrodynamic trapping are the rapid immobilization of cells compared to other trapping methods and the tools are very simple and cost-effective [12]. In comparison to the contactless methods, no advanced instrumental periphery is required. On the other hand, it is not possible to avoid contact with a surface, which could result in irreversible attachment [2]. A disadvantage is that it is hard to attain accuracy in particle deposition. Array sites often stay vacant or in place of single particles, aggregates are trapped. Therefore, strategies are being created to enhance hydrodynamic trap selectivity. Furthermore, it is of present concern to immobilize distinct kinds of an item at elevated density and to use hydrodynamic trapping ideas in specified locations [20]. So our goal is to resolve these flaws by designing and analyzing our model.

In this work, Hydrodynamic cell trapping method is used to make cells move along the microchannels and get laterally trapped in the bottom microchannel. Using Comsol Multi physics, the micro channel has been designed using CFD module, simulated and analyzed [14].

In this paper, we proposed new designs in $2 \mathrm{D}$ using Comsol and additional discussions on the simulation outcome of single cell trapping within the trap region. Single cell trapping was done by operating the rate of fluid flow and bottom channel geometry. The single cell could be trapped inside the bottom channel from the simulation outcomes, and effective designs can be identified [17, 22].

\section{GOVERNING EQUATIONS}

The fluid flow pattern may be laminar or creeping flow. The single type flow form is also referred to as Stokes flow and happens in high-speed or small geometric length scales schemes.

The Navier-stokes equations shows the motion of fluids and also as newton's second law of motion for fluids, this includes,

$\rho\left(\frac{\partial u}{\partial t}+u \cdot \nabla u\right)=-\nabla p+\nabla \cdot\left(\mu\left(\nabla u+(\nabla u)^{T}-\frac{2}{3} \mu(\nabla \cdot u) I\right)+F \quad\right.$

Where,

u shows the fluid velocity,

$\mathrm{p}$ represents the fluid pressure,

$\rho$ is the fluid density, and

$\mu$ defines the fluid dynamic viscosity.

Together with the continuity equation these above equations are always solved:

$$
\frac{\partial \rho}{\partial t}+\nabla \cdot(\rho u)=0
$$

The Navier-Stokes equations show momentum conservation, while the equation of continuity represents mass conservation.

These equations are solved for a specific range of boundary conditions (such as inlets, outlets, and walls) forecasts the speed and heaviness of the fluid in a particular geometry. The fluid velocity is specified in the fluid flow module at the inlet and the pressure at the outlet. No-slip boundary is the specified boundary conditions at the walls (i.e. Velocity is said to be zero). The amount of Reynolds, $\mathrm{Re}=\pi \mathrm{UL} / \mu$, refers to the inertial-to-viscous force ratio. It measures the flow. The number flows of Low Reynolds are laminar or creeping, while the number flows of greater Reynolds are turbulent [21]. The Navier-Stokes equation for rectangular coordinates from the de Nevers book (constant density and viscosity) is given as: 


$$
\rho \frac{D v}{D t}=\frac{\rho \partial v}{\partial t}+\rho(v \cdot \nabla v)=-\nabla \mathrm{p}+\rho g+\mu \nabla^{2} v
$$

Where the substantial time derivative is defined as

$$
\rho \frac{D v}{D t}=\frac{\rho \partial v}{\partial t}+\rho(v \cdot \nabla v)
$$

Drag force is applied to the cells located inside the microchannel during the fluidic flow through the microfluidic channel. For a constant medium density, this kind of force given to the cell is subjected to the speed of the cell movement along with the fluid and its surface region. The relationship as shown below $[8,13]$

$$
F_{d}=\frac{1}{2} \rho v^{2} C_{d} A
$$

Where $F_{d}$ shows drag force, $\rho$ defines the liquid density, $\vartheta$ is the cell velocity, $C$ and $\mathrm{d}$ represents the cell's drag coefficient and area respectively.[13]

Speed of the fluid and area of the cell can be evaluated with the help of optical observation under a microscope among the above parameters. Drag coefficient is a dimensionless parameter, depending on the geometric shape of the particle. Reynolds number is very small for a microfluidic channel, i.e.laminar flow is observed.Thus the drag coefficient was proposed to be between $0.1-0.5$ for spherical objects.

Two forces are needed to drag a particle into the microfluidic channel, first the Newtonian force and then the gravitational force. [13]

$$
F_{d}=F_{N}+F_{g}
$$

Because of the drag force, the cell must rush and push onwards. Single cell is affected with very small gravity range and can be minimized in our experimental conditions which are at exactly low Reynolds numbers. As a consequence, Newtonian force can be equated with the drag force needed to rush the particle to move along with the fluid.

$$
F_{d}=F_{N}=m a
$$

Where $F_{d}$ is the exerted force, $m$ is the mass of cell and $a$ is acceleration due to the force. The microfluidic channel is air tight during liquid flow and the pressure inside the microchannel is completely saturated.[21] As a result, the pressure applied inside the channel is spread evenly across the entire microfluidic channel. Drag force on the cell surface at this point is equivalent to the force needed to accelerate the cell. [22]

\section{DEVICE DESIGN}

We initially design three microchannels which execute the flow of water and cell efficiently inside the channel. These below microchannels have a single inlet and outlet and

multiple channel design. The size of the microchannel ranges between 100-50 micrometer as shown in Fig.1. Cell size ranges between 1-15 micrometer. Here water is considered as a buffer solution and is injected through the inlet of the channel. Particle tracing module for fluid flow is used to the compute motion of particles in a background fluid. Particle motion can be driven by drag, gravity, magnetic and acoustophoretic forces. Here drag force is used [10]. In fluid flow interfaces, physical quantities such as stress and flow rate are used, and physical characteristics such as viscosity and density are used to describe a fluid flow. The mesh configurations determine the finite element mesh resolution used to discrete the model. The finite element separates components of geometrically easy forms; it is tetrahedron in this situation. Refine the mesh to get better computational results.

The boundary conditions include selecting the design walls, the inlet, outlet, and the values of stress and speed. Predict fluid velocity and pressure for the particular design parameters. The fluid velocity is specified in the fluid flow module at the inlet and pressure at the outlet. The wall's condition is a state of no slip border. (That is, the speed is set to zero). The amount of Reynolds, $\operatorname{Re}=\pi \mathrm{UL} / \mu$, refers to the inertial-to-viscous force ratio. It measures the flow. The number flows of Low Reynolds are laminar or creeping, while the number flows of greater Reynolds are turbulent.

Table- I: Various microchannel dimensions

\begin{tabular}{|l|l|l|l|}
\hline \multicolumn{1}{|c|}{ Parameters } & \multicolumn{1}{|c|}{$\begin{array}{c}\text { Design 1 } \\
\text { Square } \\
\text { channel }\end{array}$} & $\begin{array}{c}\text { Design 2 } \\
\text { Triangular } \\
\text { channel }\end{array}$ & $\begin{array}{c}\text { Design 3 } \\
\text { Slant channel }\end{array}$ \\
\hline $\begin{array}{l}\text { Microchannel } \\
\text { width }\end{array}$ & $2000 \mu \mathrm{m}$ & $3000 \mu \mathrm{m}$ & $2000 \mu \mathrm{m}$ \\
\hline Fluid Velocity & $1 \mathrm{~m} / \mathrm{s}$ & $1 \mathrm{~m} / \mathrm{s}$ & $1 \mathrm{~m} / \mathrm{s}$ \\
\hline \multicolumn{4}{|c|}{ Particle properties } \\
\hline Density & $2200 \mathrm{~kg} / \mathrm{m}^{3}$ & $2200 \mathrm{~kg} / \mathrm{m}^{3}$ & $2200 \mathrm{~kg} / \mathrm{m}^{3}$ \\
\hline Diameter & $1 \mu \mathrm{m}$ & $1 \mu \mathrm{m}$ & $1 \mu \mathrm{m}$ \\
\hline No. of particles & 10000 & 10000 & 10000 \\
\hline
\end{tabular}

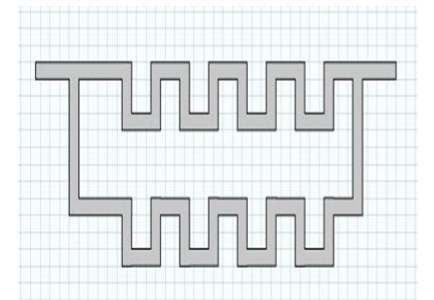

(a)

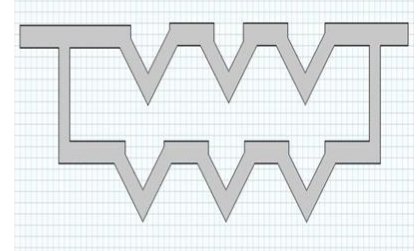

(b)

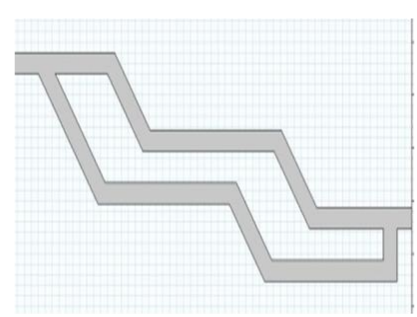

(c)

Fig. 1. Geometric objects a) Design 1- Square Shape Channel b) Design 2- Triangular Shape Channel c) Design 3- Slant channel

Published By: 


\section{SIMULATION STUDY AND RESULTS}

The comparatives study between these three channels has been done mainly to choose the better design which executes lateral trapping with optimum results.

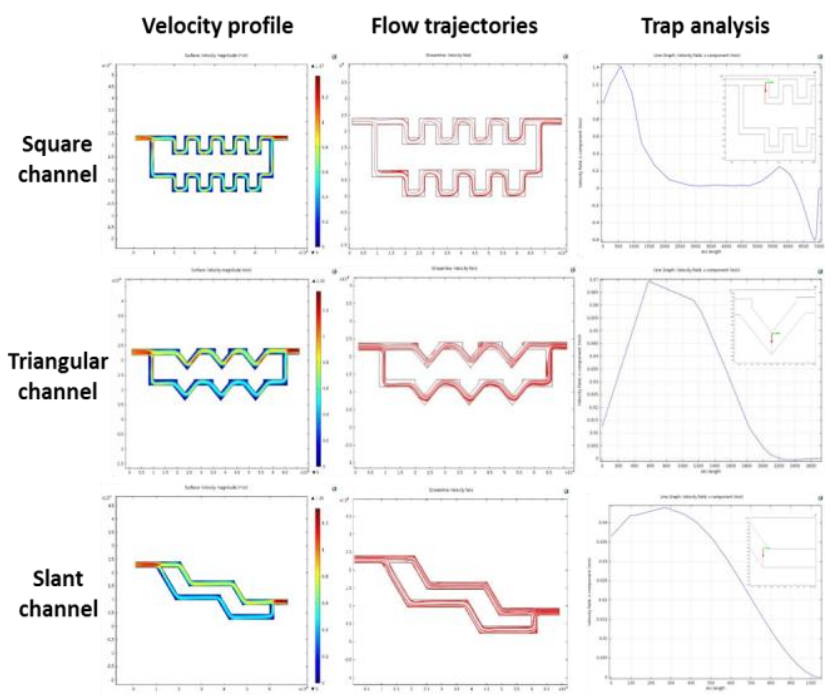

Fig. 2. Microchannel design analysis.

From the above study of channel design 1 shown in Fig.2, we can conclude that the horizontal path in the microchannel will be efficient for cell trapping when attached with traps along it. The vertical path will also leads to some cell traps. But in the triangular shape channel design there will be a high possibility of cell clumping occurs in that triangular shape channels. So trapping of a single cell is much complicated with this design. From Slant channel design, we can conclude that the horizontal path in the microchannel will be less efficient for cell trapping when attached with traps along it. The slanting path will lead to maximum cell trap, due to the high speed of the fluid at which the cell trap rate will be high. Thus the slant design is highly efficient for cell trapping laterally. Even though design 1 and 2 are efficient for attaching trap area, but there will be very less possibility for the cell to trap along the lateral traps. So we are neglecting design 1 and 2. Further enhancement of design 3 will lead to proper cell traps and optimum results. Further enhancement of channel design 2 with drain channels along with lateral trapping area

Lateral traps are attached to the sides of the microchannel as shown in Fig. 4. The cells are getting in through the inlet channel towards the main channel, the main channel consisting of trap area laterally. Also, the drain channels are attached throughout the main channel which drains out the water entering the trap area only remains the single cell in. Our design considerations are to trap individual cells in the trap region and achieve optimum outcomes. Here we are considering various channel parameters for analyses which include Slant Channel width $\left(\mathrm{SC}_{\mathrm{wt}}\right)$, Slant angle $(\theta)$, Drain Channel.

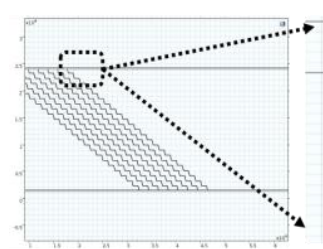

(a)

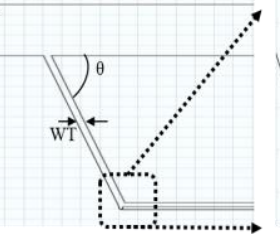

(b)

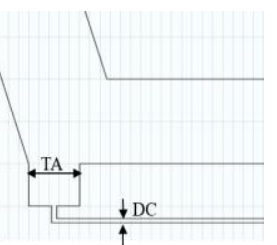

(c)
Fig. 3. Slant microchannel and its various parameters Table- II: Dimensions of the slant design

\begin{tabular}{|c|c|c|c|c|c|c|c|}
\hline $\begin{array}{l}\text { Slant } \\
\text { chan } \\
\text {-nels }\end{array}$ & $\begin{array}{c}\text { Main } \\
\text { chan } \\
\text {-nel } \\
\text { height } \\
\text { (m) }\end{array}$ & $\begin{array}{l}\text { Trap } \\
\text { area } \\
\text { width }\end{array}$ & $\begin{array}{c}\text { Drain } \\
\text { chan } \\
\text {-nel } \\
\text { width }\end{array}$ & $\begin{array}{c}\text { Slant } \\
\text { angles }\end{array}$ & $\begin{array}{c}\text { No. of } \\
\text { trap } \\
\text { area } \\
\text { in } \\
\text { each } \\
\text { channel }\end{array}$ & $\begin{array}{c}\text { Total } \\
\text { no. } \\
\text { of } \\
\text { trap } \\
\text { area }\end{array}$ & $\begin{array}{c}\text { Velo } \\
\text { city } \\
\text { rang } \\
\text { e } \\
(\mathrm{m} / \mathrm{s} \\
) \\
\end{array}$ \\
\hline SC20 & 20 & 10 & 1 & $\begin{array}{c}30^{\circ}, \\
45^{\circ}, \\
60^{\circ}, \\
75^{\circ}\end{array}$ & 23 & 184 & $\begin{array}{l}0.1, \\
1, \\
5, \\
10 \\
\end{array}$ \\
\hline SC60 & 60 & 20 & 2 & $\begin{array}{l}30^{\circ}, \\
45^{\circ}, \\
60^{\circ}, \\
75^{\circ}\end{array}$ & 27 & 216 & $\begin{array}{l}0.1, \\
1, \\
5, \\
10\end{array}$ \\
\hline SC100 & 100 & 10 & 1 & $\begin{array}{l}30^{\circ}, \\
45^{\circ}, \\
60^{\circ}, \\
75^{\circ}\end{array}$ & 31 & 248 & $\begin{array}{l}0.1, \\
1, \\
5, \\
10\end{array}$ \\
\hline
\end{tabular}

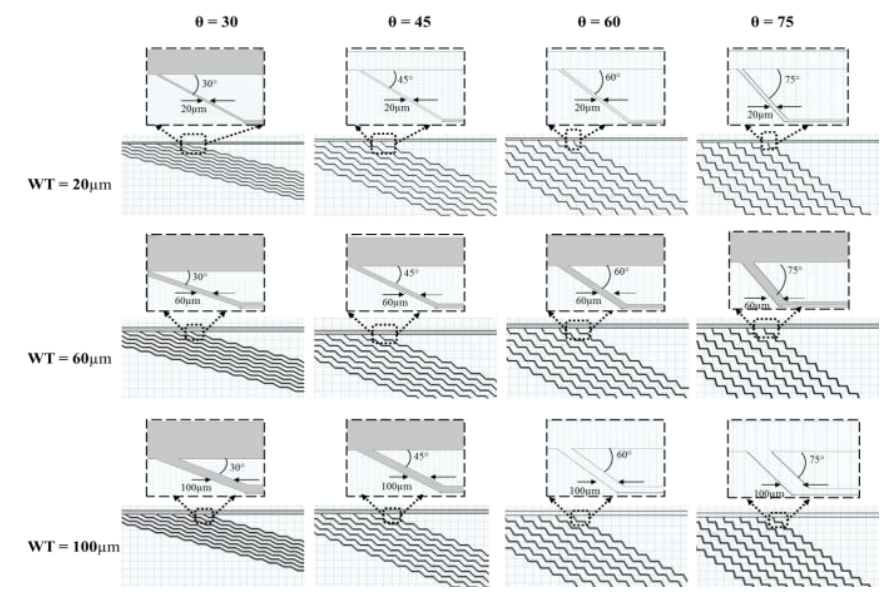

Fig. 4. Microchannel with various width $20 \mu \mathrm{m}, 60 \mu \mathrm{m}, 100$ $\mu \mathrm{m}$ with slant angles varying from $30^{\circ}, 45^{\circ}, 60^{\circ}, 75^{\circ}$

\section{A. Comparative analysis among various velocity ranges using 1D plot analyses}

These Analyses can be performed by varying the parameters of the slant design. Here the variation of the flow pattern can be studied by changing the velocity and also the variation in slant angle such as $75^{\circ}, 30^{\circ}, 45^{\circ}$ and $60^{\circ}$ respectively.

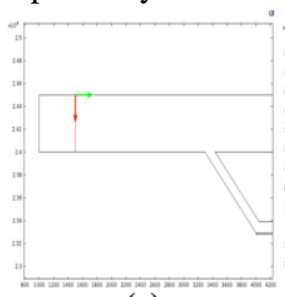

(a)

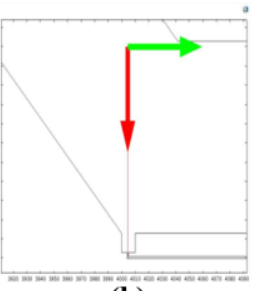

(b)

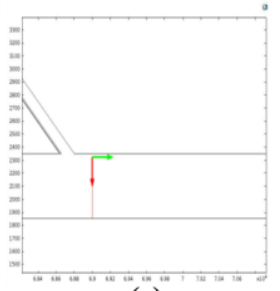

(c)
Fig. 5. Cut plane (a) At the inlet of the microchannel (b)At the trap area (c)At the outlet of the microchannel

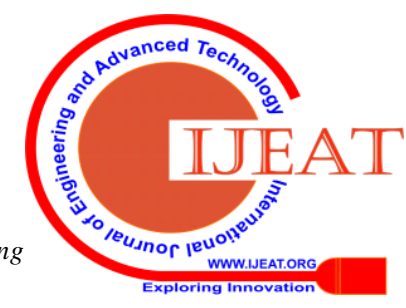


The velocity profiles show that the laminar flow is laminar [11]. If the center velocity is higher, the pressure should decrease so that the cells can stay in the centre and the remaining particles and fluid gets started to move along the focusing channel. With the simulation results shown in Fig.5, 6, 7 and 8, the possibility of the cell to get trapped inside the trap area is higher. At the inlet of the microchannel, the velocity profile for $0.1 \mathrm{~m} / \mathrm{s}, 1 \mathrm{~m} / \mathrm{s}, 5 \mathrm{~m} / \mathrm{s}$, $10 \mathrm{~m} / \mathrm{s}$ shows laminar flow is developed inside the channel. Therefore, the flow pattern is same for all the velocity range only the rate of flow varies from one another.
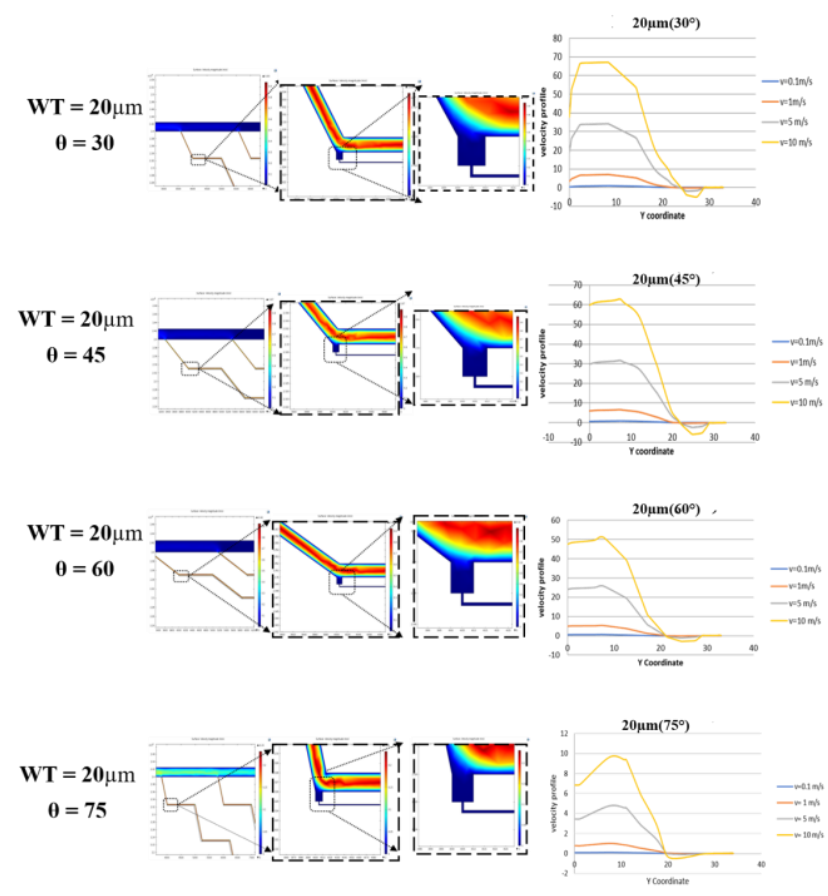

Fig. 6. Velocity profile of microchannel SC20 with various slant angles and inlet velocities

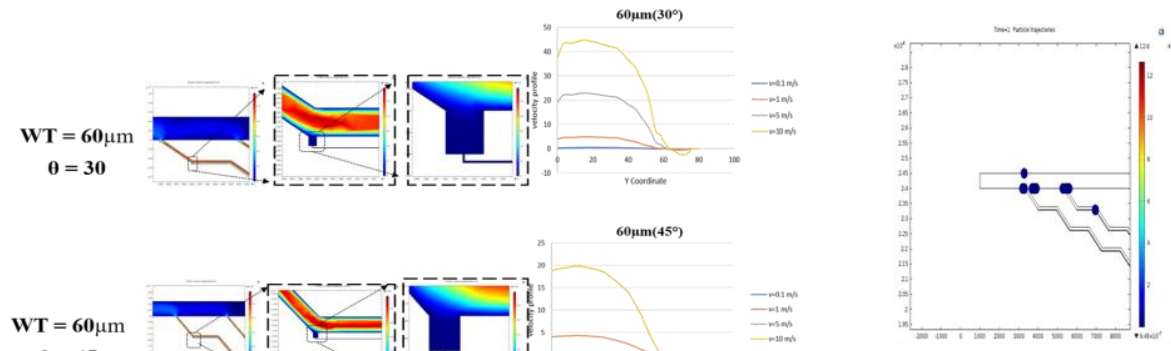

(a)

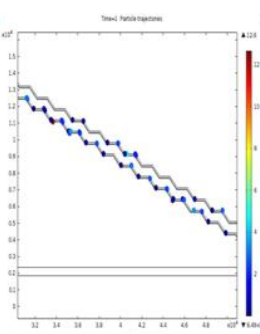

(b)

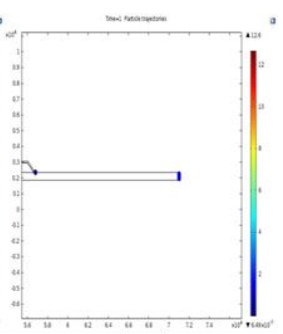

(c)
Fig. 9. (a) cell flow through the inlet channel (b) cell flow through the trap area (c) cell flow towards the outlet channel 


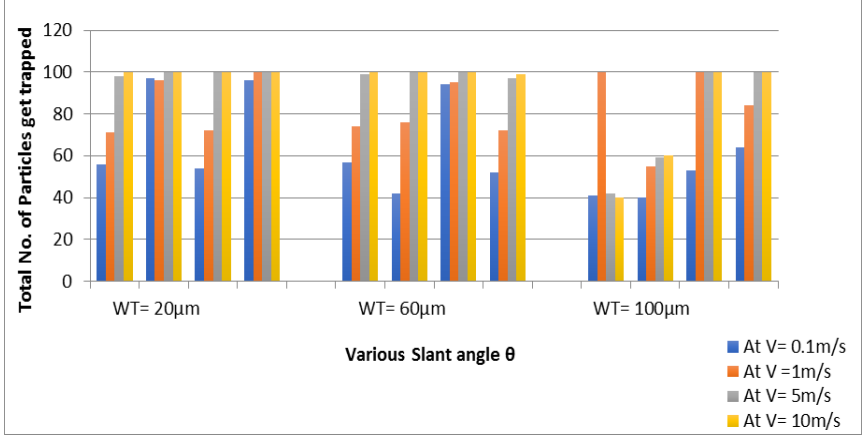

Fig. 10. Quantitative analysis of the number of particles trapped in all the channels.

\section{CONCLUSION}

From various designs, we can suggest that the slant channel design is appropriate for single cell trapping laterally. The result shows that there is more number of cells trapped inside the trap area and few of them are empty. In the slant channel design, the cell gets trapped mainly at the middle and bottom trap areas. The design also involves bottom microchannel which drains out the remaining excess water stored in the trap area to the outlet. Once the cells got trapped, the remaining cells move out through the outlet from the main channel and the drain channel.

\section{REFERENCES}

1. A computational study of single cells trapping inside a microfluidic channel Amelia Ahmad Khalili1 and Mohd Ridzuan Ahmad1 2012 IEEE EMBS.

2. Akira Yamada, Yuki Katanosaka, Satoshi Mohri, and Keiji Naruse, A Rapid Microfluidic Switching System for Analysis at the Single Cellular Level, IEEE transactions on nanobioscience, Dec 2009.

3. Amelia Ahmad Khalili, Mohd Ariffanan Mohd Basri and Mohd Ridzuan Ahmad, Single cell trapping in microfluidic channel via hydrodynamic manipulation, 2013 , IEEE International Conference on Control System.

4. Anurag Tripathi a, James Riddell, Nikos Chronis a, A Biochip with a 3D microfluidic architecture for trapping white blood cells, ELSIVIER 2013

5. Chia-Feng Liu, Jen-Yu Jao, Ming-Kun Chen, Ya-Chun Chuang, Pin-Chian Wu, and Ling-Sheng Jang, Single Cell Impedance Analysis and Electrical Characterization in Micro-fluidic Device, 6th IEEE International Conference on Nano/Micro Engineered and Molecular Systems, Feb 2011.

6. Fahmi Samsuri, Maan M Alkais, John J Evans, Biochip development using Nanoimprint Lithography (NIL) and metallic thermal evaporation techniques for biological cells manipulation using DEP, IEEE International Conference, June 2011.

7. H. kajil, s. ito, k. nagamine, m. nishizawa, n. nagai, and t. abe Characterization of retinal pigment epithelial cells and endothelial cells within a microfluidic device towards a retina on a chip, 18th international conference on miniaturized systems for chemistry and life sciences october 26-30, 2014.

8. Habibur Rahman, Member, Mohd Ridzuan Ahmad, Masaru Takeuchi, Masahiro Nakajima, Yasuhisa Hasegawa, and Toshio Fukuda, Single Cell Mass Measurement Using Drag Force InsideLab-on-Chip Microfluidics System, IEEE transactions on nanobioscience Dec 2015.

9. http://www.merriam-webster.com/dictionary/hydrodynamics

10. J. Nilsson, M. Evander, B. Hammarström, T. Laurell, Review of cell and particle trapping in microfluidic systems, Elsevier 2009.

11. Jamnani Dinesh, "Modelling and simulation of a single particle in laminar flow regime of a newtonian fluid", Proceedings of COMSOL conference 2009.

12. K. Park, L. J. Millet, N. Kim, H. Li, X. Jin, G. Popescu, N. R. Aluru, K. J. Hsia, and R. Bashir, "Measurement of adherent cell mass and growth," Proc. Natl. Acad. Sci., vol. 107, no. 48, pp. 20691-20696,Nov. 2010.

13. M. J. Fuerstman, A. Lai, M. E. Thurlow, S. S. Shevkoplyas, H. a.Stone, and G. M. Whitesides, "The pressure drop along rectangular microchannels containing bubbles," Nov. 2007
14. Norasyikin Selamat, Muhammad Syafiq Bin Rahim, Effect of Microchannel Sizes on 3D Hydrodynamic Focusing of a Microflow Cytometer, IEEE-ICSE2016 Proc. 2016, Kuala Lumpur, Malaysia.

15. Robert M. Johann Cell trapping in microfluidic chips Published online: 6 April 2006 \# Springer-Verlag 2006

16. Sukanta Bhattacharjee, Sudip Poddar, Sudip Roy, Juinn-Dar Huang, and Bhargab B. Bhattacharya, Dilution and Mixing Algorithms for Flow-Based Microfluidic Biochips, IEEE transactions on computer-aided design of integrated circuits and systems

17. S. M. Kim, S. H. Lee, and K. Y. Suh, "Cell research with physically modified microfluidic channels: A review," Lab Chip, vol. 8, Jul. 2008.

18. T. Akalin, S. Arsctt, B. Bocquet, N.E. Bourzgui, V. Mile, V. Senez, A Teizebre, Microfluidic Devices for ultra wide Band Electromagnetic Spectroscopy of Single Cell, 2006.

19. Wilfred Espulgar, Yoshinori Yamaguchi, Wataru Aoki, Daiki Mita,Masato Saito ,Jong-Kook Lee , Eiichi Tamiya, Single cell trapping and cell-cell interaction monitoring of cardiomyocytes in a designed microfluidic chip, ELSIVIER 2015.

20. Vigneswaran Narayanamurthy, S.N., Al'aina Yuhainis Firus Khan, Fahmi Samsuri, T.M.Sridhar, "Microfluidic Hydrodynamic trapping for single cell analysis: Mechanisms, Methods and Applications, Analytical Methods, 2017.

21. Narayanamurthy, Vigneswaran, Tze Lee, Al'aina Khan, Fahmi Samsuri, Khairudin Mohamed, Hairul Hamzah, and Madia Baharom. "Pipette Petri Dish Single-Cell Trapping (PP-SCT) in Microfluidic Platforms: A Passive Hydrodynamic Technique." Fluids 3, no. 3 (2018): 51.

22. James Jasper, M. Sushmitha, R. Premkumar, Vigneswaran Narayanamurthy, and R. Kalpana. "Microfluidic micro-well (size and shape) by numerical optimization for single cell applications: Vertical trapping approach." In 2017 International conference on Microelectronic Devices, Circuits and Systems (ICMDCS), pp. 1-6. IEEE, 2017.

\section{AUTHORS PROFILE}

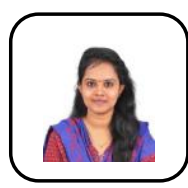

Ms. M. Sushmitha is currently working in the Department of Biomedical Engineering, Kalasalingam Academy of Research and Education. She had completed her Graduation, B.E in Engineering from Rajalakshmi Engineering College and Post-Graduation in Medical Electronics from Rajalakshmi Engineering College. She has published her research papers in various IEEE international conferences and one journal.

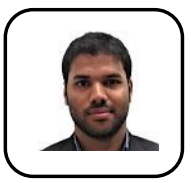

Dr. Vigneswaran Narayanamurthy is an academician cum researcher with several years of experience in research, teaching and consultancy in the field of Electronics and Biomedical Engineering. Currently he is serving as senior lecturer in the faculty of electrical and electronic engineering technology, Universiti Teknikal Malaysia Melaka. Have published in several indexed international journals and holds two patents. Products developed through past research include a passive lab on chip applications, expert systems, and agriculture technologies.

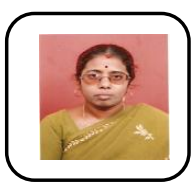

Padmasini $\mathbf{N}$ is currently working in the Department of Biomedical Engineering, Rajalakshmi Engineering College. She had completed her Graduation, B.E in Electronics and Communication Engineering from Madurai Kamaraj University and Post Graduation in Biomedical Engineering from Anna University. She is currently pursuing her research in the topic of SDOCT Retinal Images under Anna University. She has published her research papers in three IEEE international conferences, three book chapters and two journals. 